\title{
Documents en action dans une organisation : des négociations à plusieurs niveaux
}

Documents for Action in Organizations: The Case of Multilevel Negotiations

Sylvie Dalbin et Brigitte Guyot

\section{(2) OpenEdition}

Journals

Édition électronique

URL : http://journals.openedition.org/edc/467

DOI : $10.4000 /$ edc. 467

ISSN : 2101-0366

Éditeur

Université Lille-3

Édition imprimée

Date de publication : 1 octobre 2007

Pagination : 55-70

ISBN : 978-2-9514961-9-4

ISSN : $1270-6841$

Référence électronique

Sylvie Dalbin et Brigitte Guyot, "Documents en action dans une organisation : des négociations à plusieurs niveaux ", Études de communication [En ligne], 30 | 2007, mis en ligne le 01 octobre 2009, consulté le 01 mai 2019. URL : http://journals.openedition.org/edc/467 ; DOI : 10.4000/edc.467

Ce document a été généré automatiquement le 1 mai 2019.

(c) Tous droits réservés 


\title{
Documents en action dans une organisation : des négociations à plusieurs niveaux
}

\author{
Documents for Action in Organizations: The Case of Multilevel Negotiations
}

\author{
Sylvie Dalbin et Brigitte Guyot
}

1 L'approche communicationnelle des organisations ${ }^{1}$ prend sous son regard des objets médiateurs utilisés en leur sein pour étudier la dynamique des acteurs autour d'eux : ce sera le cas des documents produits et utilisés en entreprise. Elle pose comme fondamentale la relation entre document et action [Guyot, 2006], reconnaissant que celuici à la fois forme et est formé par les interactions sociales que suscite une activité professionnelle. Ces échanges se sont longtemps réalisés au moyen de supports matériels analogiques comme le papier qui offrait un support autonome rendant déjà possibles des interactions éloignées dans le temps et dans l'espace [Goody, 1977]. Aujourd'hui, que deviennent-elles avec le passage au numérique? Nous voudrions montrer qu'une étude attentive des conditions de production, de gestion/organisation et d'utilisation de documents laisse voir des espaces de négociation qui impactent la forme même du document ainsi que son utilisabilité [Le Coadic, 1997 ; Tricot, 2001].

2 La littérature concernant le document constitue un espace pluridisciplinaire dont les textes de Pédauque se sont fait la synthèse, qui distingue trois approches spécifiques mais en interaction: (i) le document est un espace d'inscription de signes, selon des grammaires et des langages conventionnels pouvant donner lieu à des calculs ; (ii) c'est un espace de sens qu'il convient d'appareiller avec des marquages destinés à en faciliter l'accès et l'exploitation par le public cible; (iii) enfin, le document crée un espace de relations sociales [Pédauque, 2006].

3 En reprenant ce cadre, nous montrerons le document pris dans un réseau d'acteurs et de négociations (iii), qui se transforme face à son équivalent analogique, à condition de faire évoluer à la fois l'espace des signes (i) et celui des grammaires et langages adaptés aux 
différents besoins/pratiques professionnelles, par le biais d'une plus grande structuration, d'un enrichissement des clés d'accès ou d'outils d'annotation.

4 D'autres auteurs ont relevé les difficultés liées à la production documentaire, en particulier les documents techniques et ceux qui sont prescripteurs [Leplat, 1997, 2004], la difficulté étant de les adapter à plusieurs cibles et par conséquent de les structurer de sorte que chacun y trouve son compte. Or contraintes, conflits d'intérêts, poids des hiérarchies ou encore pressions contradictoires des acteurs eux-mêmes viennent freiner cette structuration.

5 Quittant le territoire documentaire pour nous placer sur celui de l'action, nous parlerons de document en actionpour indiquer le rapport étroit entre l'action, l'organisation du travail et le pouvoir des acteurs. Les modèles d'organisation par projet montrent différentes approches: approches traditionnelles planificatrices (les acteurs sont soumis à des contraintes et à un pouvoir coercitif), économiques (on parle alors de fournisseur et de client), approches plus contemporaines de type coopératif plutôt orientées vers une gestion des opportunités et une réduction des contraintes [Cornu, 2004; Staquet, 2005] ou encore approche de type intelligence cognitive basée sur la co-construction de référentiels de connaissance [Ballay, 1997]. Chacune d'elle détermine des rôles et des pouvoirs de négociation variables qui rejaillissent sur les territoires documentaires [Belin, 2006].

6 Après avoir défini ce qui constitue l'espace documentaire comme espace d'interactions multiples, nous voudrions montrer que s'y exercent des négociations tant sur la forme que sur l'exploitation des contenus, et démontrer qu'une forme traditionnelle imprègne encore largement la conception et l'usage des documents numériques, les usages ou l'exploitation liés au numérique ayant du mal à être intégrés dans une organisation.

7 Par document en action nous faisons référence aux documents porteurs d'informations liées directement à l'action, la sienne ou celle d'autrui [Guyot, 2006; Zacklad, 2005], comptes-rendus de réunion, d'expériences, de visite, cahiers des charges, rapports techniques, manuels de procédures. Nous suivons la définition du document numérique, selon laquelle des données sont enregistrées sur un support, assorties d'une forme, d'une signification, servant à la consultation, à l'étude, à la preuve ou à la trace. Cette unité autonome nécessite un dispositif pour être lue et exploitée. Cette définition fait d'une base de données ${ }^{2}$ ou d'information, un document.

8 Et puisqu'il s'agit de communiquer un message encapsulé dans un document par un auteur/émetteur à destination d'un lecteur/récepteur, le choix du terme d'acteur semble pertinent pour indiquer un agent actif qui endosse possiblement plusieurs rôles, celui d'auteur lorsqu'il écrit, d'éditeur quand il met en forme son texte ou celui d'un autre, et bien évidemment celui de lecteur.

9 Le rôle d'éditeur prend alors ici un sens fort. En effet, il ne suffit pas d'imposer à un auteur une forme pour guider sa rédaction (comme le font un formulaire ou un modèle de document) pour qu'il se transforme en éditeur. Celui-ci agit aussi sur la structure et sur les modèles de documents afin d'en optimiser la circulation dans des espaces de travail ou collectifs multiples. Il y a donc deux niveaux, l'un conceptuel (mise en sens et en intelligibilité, à partir de modèles de données métiers) et l'autre formel (matrices réexploitables, feuilles de style et formats XML avec balisage), qui construisent un usage par anticipation. Qui est alors l'acteur légitime pour réaliser ce travail de répartition/ organisation? Cette transformation de la matière première du document est un travail 
documentaire qui le charge d'éléments permettant de le retrouver ou d'indiquer le type d'action possible, ce qui implique de distinguer entre ce qui relève d'une production pour son propre compte, inhérent à toute activité de travail (que nous appellerons modèle auteur), et ce qui relève d'un travail réalisé d'autres que soi, ce qui le propre de l'activité de prestataire (modèle de service).

\section{Espaces d'interaction documentaire et négociations}

Commençons par préciser les deux grandes familles d'espaces documentaires qui influencent l'activité liée au document en nous aidant de la distinction modèle auteur et modèle de service. Elle nous permettra de cerner la complexité de ce qui intervient dans la circulation documentaire.

\section{Produire pour soi : le modèle auteur}

11 Une première famille correspond aux espaces formés par l'activité de travail d'un individu ou d'un groupe d'individus. Celle-ci convoque des gestes, mobilise des ressources d'ordre matériel (équipements), humain ou immatériel (règles, normes, informations et documents variés) [Leplat, 2004]. L'activité regroupe des façons de faire, des manières d'être en relation avec les autres. Elle engage également une activité d'information, soit pour en produire (auteur) soit pour l'exploiter (lecteur), les tâches de production et d'exploitation étant parfois intimement mêlées.

À regarder de plus près cet espace d'activité, on voit poindre ce que nous appelons une activité d'information [Guyot, 2007] au cœur même de l'activité. En effet, une partie croissante de celle-ci est consacrée à dire ce qu'on fait, à rendre compte des actions réalisées, et à s'informer sur ce que font les autres. Selon les moments, la même personne sera amenée à se comporter en auteur, en utilisateur et en gestionnaire de ses propres dossiers personnels, lesquels, de plus en plus fréquemment, deviennent des dossiers collectifs lorsqu'ils sont mis à disposition de collègues et a fortiori quand ils seront coproduits avec eux. C'est ainsi qu'on peut parler de chaîne éditoriale, pour décrire la façon d'élaborer des documents et de les mettre en circulation [Kolmayer, Peyrelong, 1999]. Nous parlerons d'espace documentaire pour indiquer le contexte, le mode d'organisation, le type d'activité et le type d'acteur (chef d'atelier, directeur d'exploitation, chercheur ou commercial, décideur), et le degré de formalisation et d'obligation. Le déploiement des principes qualité et sécurité, et plus récemment, ceux qui sont liés à l'optimisation des activités ont impacté cet espace et contribué à le (re)structurer. C'est le cas des modèles de comptes-rendus ou de retours d'expérience qui doivent être suffisamment formalisés et explicites pour que ceux qui n'ont pas participé à leur élaboration puissent les comprendre. Un certain nombre d'outils visent à réduire les risques d'incompréhension, tels les référentiels communs, réalisés en dehors des espaces de travail individuels.

13 Selon les secteurs ou les types d'activité, cet espace d'interaction documentaire peut s'avérer plus ou moins complexe car centré sur d'autres auteurs/partenaires proches, ou bien être étendu à d'autres familles d'acteurs. C'est l'un des paradoxes actuels : l'exigence s'accroît d'imbriquer fortement information et action (par contextualisation), alors même qu'on demande à l'auteur/producteur de s'abstraire de ses contraintes propres pour prendre en compte ou entrer dans la logique de pensée d'un autre [Thévenot, 2006]. Cela pousse, en tout cas, à réfléchir aux implications de ce type de discours. 


\section{Produire pour autrui : le modèle de service} d'être stabilisés par une décision: les modalités peuvent varier du dialogue visant un accord entre les parties, à l'imposition d'un point de vue final dominant. Utiliser ce terme à propos du document, c'est relever la présence de nombreux acteurs concernés par la production et l'exploitation d'information et insister sur leur capacité d'expression et de discussion. Des accords se réalisent, des conflits d'intérêt se résolvent ou perdurent, dont le document portera les marques dans sa structure même.

montée des négociations est due, pour l'essentiel, au fait de travailler en mode projet, ce travail collectif ayant comme conséquence que tout travailleur peut devenir le prestataire d'un autre. À partir du moment où l'on porte attention à des documents censés être valables non plus pour soi mais pour un service ou un groupe de personnes, les terrains de négociation s'élargissent [Thévenot, 1997].

Une première conséquencetouche le statut traditionnel d'auteur (écrire personnellement, en son nom ou pour d'autres). Le constat d'un déplacement vers un certain anonymat ${ }^{3}$ au profit de contributeurs pluriels qui interviennent sur le document - rédacteurs, valideurs, référents, peut aboutir à une signature collective ${ }^{4}:$ il devient alors difficile de distinguer le producteur de l'information (l'auteur?), de l'éditeur prestataire de service. 
21 La seconde conséquencefait apparaître un rôle nouveau qui consiste à ajouter du para-texte ou marquages documentaires au texte initial. Les contraintes juridiques poussent à rendre visibles ce rôle documentaire. La troisième conséquence, celle qui sera développée ici, porte sur la forme du document en tant qu'elle résulte et donc reflète ces négociations et, par là même, le poids des différents acteurs qui y ont été impliqués.

\section{Le document crée un espace de sens qui anticipe une action potentielle}

23 En même temps qu'un espace d'inscription, le document porte un potentiel d'action dans la mesure où il rassemble à sa surface des informations appartenant à des systèmes différents. Jeanneret a montré combien, dans un écrit d'écran, interfèrent plusieurs couches, technique, cognitive et sociale: contenu agencé et rendu disponible par des normes techniques, logicielles, ergonomiques et éditoriales qui en font un espace d'appréhension et d'exploitation dans et avec lequel l'utilisateur interagit [Jeanneret, 2000]. Le document engrange des usages potentiels plus ou moins déterminés d'avance.

activité éditoriale consiste alors à relier les éléments constitutifs d'un document à une action par un travail de décomposition/recomposition. Un compte-rendu de réunion, par exemple, apporte plusieurs sortes d'information, chacune renvoyant à une action possible : la décision de lancer un projet, le mode de financement ou encore les tâches à assurer, chacune impliquant des services (ou acteurs) différents. S'il pouvait intégrer les usages dans sa structure même et ne fournir que ce qui concerne un acteur, ce compterendu ainsi transformé, associerait une équipe à chaque point traité. Les outils actuels permettent de ne diffuser que la partie concernant un point précis au responsable désigné, en rappelant la source (la réunion la date, les participants, c'est-à-dire les éléments permettant de l'identifier).

C'est l'une des principales évolutions qui sépare un document linéaire et une banque de données structurée ${ }^{5}$. Plutôt que de suivre l'ordre des questions abordées un compterendu structuré par appariement d'informations et d'usages est devenu interrogeable pour une action précise et ne fournit que ce qui la concerne ${ }^{6}$. On voit aussi qu'un tel travail est d'ordre managérial, alors que souvent il est considéré comme une simple question technique.

Force est de constater qu'aujourd'hui ce type de document est encore mis en circulation en entier sans ce travail de découpage en unités d'information significatives destinées à telle ou telle entité. Cela signale un déficit d'analyse sur l'adéquation entre l'information et l'action, un document restant encore considéré comme une unité insécable et non comme un réservoir d'informations immédiatement exploitables.

Le diffuser tout entier, c'est suivre une orientation producteur-auteur, celui-ci se valorisant à travers cette production globale, alors qu'isoler les différentes unités d'information pour les articuler avec les espaces documentaires (les intégrer à une base de données), serait suivre une logique utilisateur associée à une action. 


\section{Deux exemples de documents négociés}

\section{Documents utiles à une activité d'analyse documentaire}

maintenir : ils ne sont pas toujours utilisables, du fait que les besoins qui émergent au fil de l'activité d'analyse sont mal pris en compte; ils ne sont pas toujours adaptés à une recherche qui sort de la routine (les règles à appliquer pour un site web diffèrent de celles définies pour un retour d'expérience). C'est ainsi que s'il existe une charte stratégique, le manuel de référence fait souvent défaut, ou bien il est sous-exploité ou peu mis à jour. Ces jeux de présence ou d'absence de tel ou tel document indique les espaces de négociations possibles. Ces questions de planification documentaire sont connues des rédacteurs techniques chargés de produire manuels ou guides. Sans aller jusqu'à réduire cette production documentaire à un document unique qui regrouperait toutes les données utiles sous une forme lisible, il pourrait sembler intéressant de rapprocher certains documents, au risque d'accroître le nombre de conflits : par exemple sur les informations à regrouper (méthode d'analyse, règles), leur degré de précision et leur description suivie ou non d'exemples, l'organisation de ces informations entre elles, le cadre pour les structurer, l'ordre et le style rédactionnel à adopter, la récupération de ces informations pour l'aide contextuelle des applications informatisées...

Or construire une base de connaissance pour remplacer la forme traditionnelle, offrirait une plus grande souplesse car ce lot de documents, lourds à maintenir et difficile à utiliser, serait remplacé par un système d'information unique. À ces documents autonomes se substituerait un corpus à partir duquel il serait possible d'éditer, si besoin, des documents qui peuvent être ceux d'aujourd'hui, mais aussi d'autoriser les analystes à commenter, annoter ou à poser des questions à leurs collègues. Mais une telle initiative semble poser problème. Malgré leur sous-utilisation, les documents actuels constituent une preuve tangible (un vrai document, nous dit-on) de l'intérêt que chacun porte à 
l'activité d'analyste. Peut-on en conclure que les enjeux sociaux importent davantage que l'utilité d'un document?

\section{Direction R\&D d'une entreprise industrielle}

De nombreux secteurs industriels et scientifiques ont commencé à améliorer la diffusion de la documentation liée à leurs activités, ensuite sa qualité et sa pérennisation, et, plus récemment, sa valorisation et sa ré-exploitation par le biais d'une capitalisation et d'un enrichissement [Ballay, 1997]. Le tableau synthétique suivant montre l'arrivée d'une nouvelle famille d'acteurs à côté des auteurs traditionnels et des instances dirigeantes, celle des contributeurs ajoutant des éléments de données aux documents.

Initialement ces documents se composaient de rapports de recherche ou d'études. Les premières pages procurent des informations descriptives et contextuelles (auteur/ affiliation, date, résumé/mots clés, nom du projet), zone qui a été considérablement enrichie et structurée.

$\mathrm{Au}$ fil du temps pour prendre en compte les besoins de différentes catégories d'acteurs: gestion du document lui-même (clarification du rôle d'auteur, statut du document), gestion de la diffusion du document source ou de sa synthèse, gestion de sa conservation (lieu, durée d'archivage), contextualisation (nom du projet ou de l'affaire, voire étape du projet à laquelle se rattache le document), sécurité (droit d'accès, niveau et durée de confidentialité), qualité (en référence à une norme).

Dans ces rapports se retrouve la macrostructure propre au discours scientifique: contexte et environnement de l'étude, méthode et expérimentation, résultats, discussion. Le développement de l'assurance-qualité a contribué à élaborer des macrostructures adaptées à d'autres types de documents comme les cahiers des charges ou des relevés d'expériences.

36 Ainsi, avec le développement des modalités de traitement de l'information, le nombre d'acteurs impliqués dans les espaces se multiplie dans une grande diversité de profils. Après la gestion du document, est venue la valorisation de son contenu, et aujourd'hui son enrichissement par un paratexte qui impacte directement le contenu et ses conditions de production. L'intégration d'hypertexte ainsi que des outils numériques devrait encore faire évoluer ces espaces documentaires.

Par contre si la nature des interactions dans ces espaces ne semble pas avoir été affectée par la numérisation, les traces qu'elles laissent sur le document diffèrent de celles du monde analogique: structuration plus forte et, surtout, formalisme plus poussé pouvant aller jusqu'à supprimer le document traditionnel pour le transformer en bases de données. Dans ce contexte, on reste étonné que certains outils qui organisent l'accès à l'information ou la navigation restent inchangés (sommaire, index de fin de livre, plan de classement).

Car en définitive, cet espace documentaire et d'interaction apparait paradoxal. On le voit s'élargir, du fait qu'un document concerne potentiellement de nouveaux acteurs amenés à négocier ou à renégocier, tout comme l'accroissement du nombre de documents et leur variété formelle. Et pourtant, cet espace se restreint car la multiplication des préformatages entraîne une certaine stabilisation qui réduit d'autant les occasions de négocier. 


\begin{tabular}{|c|c|c|c|}
\hline $\begin{array}{l}\grave{A} \\
\text { partir } \\
\text { de }\end{array}$ & Forme finale du document & $\begin{array}{l}\text { Besoin nouveau et } \\
\text { action modificatrice sur } \\
\text { le document }\end{array}$ & Acteurs concernés \\
\hline \multirow[t]{2}{*}{1980} & $\begin{array}{l}\text { Document papier autonome. } \\
\text { Diffusion de notice ou du document } \\
\text { entier }\end{array}$ & $\begin{array}{l}\text { Diffusion personnalisée } \\
\text { pour alléger l'émission } \\
\text { de documents }\end{array}$ & $\begin{array}{l}\text { Auteurs/ } \\
\text { Informatique } \\
\text { Archives, } \\
\text { documentation }\end{array}$ \\
\hline & $\begin{array}{l}\text { Document papier ou numérique } \\
\text { (pdf), autonome, intégrant une } \\
\text { synthèse, venant compléter les } \\
\text { résumés d'auteurs }\end{array}$ & $\begin{array}{l}\text { Forme adaptée au profil } \\
\text { d'utilisateur : la } \\
\text { direction reçoit } \\
\text { uniquement la synthèse }\end{array}$ & Auteurs/Direction \\
\hline 1985 & $\begin{array}{l}\text { Document papier ou numérique } \\
\text { (pdf), intégrant des données liées à la } \\
\text { gestion de projet ou la gestion } \\
\text { qualité }\end{array}$ & $\begin{array}{l}\text { Suivi des règles qualité } \\
\text { - ISO }\end{array}$ & $\begin{array}{l}\text { Auteurs/Direction/ } \\
\text { Qualité }\end{array}$ \\
\hline 1990 & $\begin{array}{l}\text { Documents numériques (pdf) : } \\
\text { construction de liens formalisés, } \\
\text { métadonnées entre documents }\end{array}$ & $\begin{array}{l}\text { Gestion de la } \\
\text { documentation liée à } \\
\text { des projets }\end{array}$ & $\begin{array}{l}\text { Auteurs/Gestion de } \\
\text { l'information/ } \\
\text { records management }\end{array}$ \\
\hline 1990 & $\begin{array}{l}\text { Pour certains documents (relevés des } \\
\text { mesures d'expériences), transferts } \\
\text { vers des bases d'information }\end{array}$ & $\begin{array}{lr}\text { Conception } & \text { de } \\
\text { documents } & \text { bases } \\
\text { d'information } & \end{array}$ & $\begin{array}{l}\text { Auteurs/Gestion des } \\
\text { connaissances }\end{array}$ \\
\hline $\begin{array}{l}\text { Depuis } \\
\text { fin } \\
1990\end{array}$ & $\begin{array}{l}\text { Développement de systèmes } \\
\text { documentaires spécialisés (gestion de } \\
\text { projet, record management, gestion } \\
\text { qualité...) et de portail personnel du } \\
\text { collaborateur. Le document se } \\
\text { reconstruit en fonction du profil de } \\
\text { l'utilisateur - les 1ères pages } \\
\text { deviennent } \\
\text { documentaire en soi : un méta- } \\
\text { document }{ }^{7} \\
\text { - document créé dynamiquement (= } \\
\text { virtuel) }\end{array}$ & $\begin{array}{l}\text { Optimiser temps et } \\
\text { moyens de traitement } \\
\text { meilleure réutilisation } \\
\text { de l'information, quelle } \\
\text { que soit sa forme et son } \\
\text { support }\end{array}$ & $\begin{array}{l}\text { Tous les acteurs } \\
\text { concernés par } \\
\text { l'information et les } \\
\text { documents Tous les } \\
\text { acteurs, y compris les } \\
\text { lecteurs }\end{array}$ \\
\hline
\end{tabular}

Évolution des espaces de négociation

\section{De quelques malentendus}

Un certain nombre de malentendus peuvent être directement lié à un défaut de négociation. Tout d'abord, cette activité documentaire anticipant l'utilisation reste largement implicite alors même qu'elle devient largement distribuée : entre les acteurs responsables de la conception et des utilisateurs à qui l'on confie certaines tâches de production, d'annotation ou de validation. Or toute négociation prend du temps car elle 
suppose que les avis s'expriment que les besoins adaptés à toutes les situations soient pris en compte. Ces malentendus sont d'ordre organisationnel, quand une co-production de documents se heurte aux cloisonnements entre catégories de personnels (hiérarchie) et entre métiers (documentalistes/utilisateurs ou producteurs/utilisateurs) et en raison des conséquences que ces co-activités ont sur les pratiques de travail. Une production coopérative plus intense conduit à des renonciations, des accords sur les informations, leur forme et leur regroupement. Les discussions se complexifient d'autant plus que le nombre d'acteurs s'accroît et qu'augmentent les critères sur lesquels portent les concessions nécessaires. Si, jusque-là, les négociations portaient sur la forme finale, le travail en réseau et à distance (par le biais d'outils de rédaction/co-rédaction tels les wikis) impose une organisation, des règles et des outils, ce qui suppose de mieux prendre en compte les processus et non seulement les résultats.

C'est dire que le renforcement du rôle éditorial pousse à clarifier les rôles de chacun, quand ce n'est dégager du temps pour mettre les acteurs ensemble et les pousser à s'entendre sur des règles qu'ils puissent ensuite respecter. Cette insertion d'une attention documentaire dans sa propre activité de travail ou dans celle des autres est encore peu accompagnée sur le plan managérial.

Des malentendus d'ordre technique se remarquent, enfin, lorsqu'on continue à travailler avec des modèles de documents qui n'intègrent pas les apports du numérique ${ }^{8}$ comme les outils d'annotation dédiés, utiles au simple lecteur et pas seulement à l'auteur/rédacteur.

Il nous semble que, si l'on veut que le numérique devienne une réelle aide au travail quotidien, il paraît indispensable de comprendre cette dynamique éditoriale et documentaire afin de déterminer les compétences qu'implique la variété des rôles qu'un acteur est amené à endosser, ce qui est bien l'une des marques du travail aujourd'hui.

\section{BIBLIOGRAPHIE}

Ballay, J.-F., (1997), « Capitalisation collective du savoir-faire : l'expérience du projet Diadème

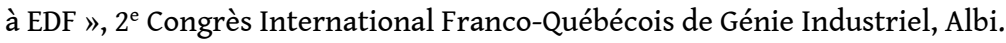

Belin, O. et Morillon, L., (2006), «L'intranet comme révélateur des libertés : jeux, pouvoirs et stratégies d'acteurs dans les organisations ", Colloque international Pratiques et usages organisationnels des Sciences et TIC, Université de Rennes.

Broudoux, E., (2005), « Construction de l'auteur autour de ses modes d'écriture et de publication », http://archivesic.ccsd.cnrs.fr/sic_00001552.html.

Cornu, J.-M., (2004), « La coopération, nouvelles approches », http://www.cornu.eu.org/texts/ cooperation.

Chartron, G. et Guyot et alii, (2002), « Le document numérique : un objet fédérateur en sciences de l'information, Documentaliste Sciences de l'information », vol. 39, n 6, pp. 298-305.

Goody, J., (1977), La raison graphique, de Minuit (éd.).

Guyot, B., (2006), Dynamiques informationnelles dans les organisations, Hermès-Lavoisier. 
Guyot, B., (2007), « Processus éditorial : faire passer un document d'un monde à l'autre », Où va le travail à l'ère du numérique?, A.-F. de saint Laurent-Kogan et J.-L. Metzger (éds.), Mines de Paris, Presses ParisTech.

Jeanneret, Y., (2004), « Forme, pratique et pouvoir. Réflexions sur le cas de l'écriture », Sciences de la société, $\mathrm{n}^{\circ} 63$.

Jeanneret, Y., (2000), Y a-t-il (vraiment) des technologies de l'information ?, Lille, du septentrion (éd.).

Kolmayer, E. et Peyrelong, M.-F., (1999), « Partage de connaissances ou partage de documents? ", Document Numérique, n 3-4, pp. 283-299.

Leplat, J., (1997), Regards sur l'activité en sitation de travail, P.U.F.

Leplat, J., (2004), « Procédures d'objectivation dans un entretien de recherche », @ctivités, 1 (2), 195-216, http://www.activites.org/v1n2/Leplat.pdf.

Pédauque, R. T., (2006), Le Document à la lumière du numérique : Forme, texte, médium : comprendre le rôle du document numérique dans l'émergence d'une nouvelle modernité, CF éditions.

Pédauque, R. T., (2007), La Redocumentarisation du monde, Cépadues.

Rohmdane, M. B. et Lainé-Cruzel, S., (1997), « Prise en compte de la structure des articles en sciences agronomiques pour la navigation dans un corpus scientifique électronique », journées SFSIC-ENSSIB.

Staquet, C., (2005), « Qu'est-ce qui différencie la collaboration de la coopération ? ", Animation \& Éducation, $\mathrm{n}^{\circ} 187 / 188$.

Thévenot, L., (1997), « le gouvernement par les normes Formes de qualification des biens et des personnes : normalisation, certification $»$, Raisons pratiques, $\mathrm{n}^{\circ} 8$.

Thévenot, L., (2006), L'action au pluriel, La découverte (éd.).

Tricot, A., (2001), « Interpréter les liens entre utilisabilité et utilité des documents électroniques ", M. Mojahid \& J. Virbel (éds.), Les documents électroniques, méthodes, démarches et techniques cognitives, Paris, Europia.

Zacklad, M., (2005), « Processus de documentarisation dans les Documents pour l'Action (DopA) : statut des annotations et technologies de la coopération associées ", http:// archivesic.ccsd.cnrs.fr/index.php? halsid=72884d028942e88eef7c95f0c88d987f\&view_this_doc=sic_00001072\&version=2.

\section{NOTES}

1. Entendu comme un système social qui se structure autour d'un but.

2. Bases de données ou d'information, constituent effectivement un contenant regroupant de façon cohérente des données (factuelles) utilisables comme ressource d'information (observatoires statistiques, contrathèques, bases des décision).

3. Certains auteurs vont jusqu'à évoquer l'effacement de l'auteur [Broudoux, 2005].

4. Sous l'auteur Pedauque se cachent de multiples contributions rédigées par un ensemble d'auteurs appartenant au groupe RTP document, R. Pédauque en étant la forme autorisée.

5. Le premier est analogique, même s'il prend la forme numérique; rappelons aussi que nous considérons le second comme un document.

6. Champs représentés aujourd'hui par les balises au sein d'un document numérique XML. 
7. Éventuellement porté dans un contenant de type base de données.

8. Par exemple, la majorité des documents au format PDF sont produits sans signets ni table des matières...

\section{RÉSUMÉS}

L'approche communicationnelle des organisations étudie la dynamique des acteurs autour d'objets médiateurs comme les documents, et pose comme fondamentale la relation entre une action et un document. Les auteures montrent comment des négociations entre les différents acteurs en présence (auteurs, éditeurs, gestionnaires et plus récemment lecteurs) impactent la forme et l'utilisabilité des documents. Deux exemples (documentation de référence pour indexeurs, rapports techniques) illustrent cette position. En conclusion, les auteurs rappellent quelques malentendus d'ordre organisationnel, managérial ou technique, propres à cette situation.

A communication approach to organizations analyzes social dynamics as mediator objects such as documents and stresses the relationships linking actors and documents. This paper shows how negotiations between stakeholders (authors, publishers, managers, and more recently readers) impact the form and usability of documents. Two cases (reference documentation used by index compilers and technical reports) illustrate this position. In conclusion, the paper recalls a number of organizational, managerial, and technical misunderstandings related to these situations.

\section{INDEX}

Keywords : digital document, work document, publishing, organizational communication, espace documentaire, redocumentarization

Mots-clés : document numérique, document de travail, édition, communication organisationnelle, espace documentaire, redocumentarisation

\section{AUTEURS}

\section{SYLVIE DALBIN}

Sylvie Dalbin est consultante en organisation et ingénierie documentaires depuis 1989 au sein d'Assistance \& Techniques Documentaires. Elle conduit auprès des entreprises et organismes, des actions de conseil et d'accompagnement centrées sur l'architecture des systèmes documentaires en vue de valoriser l'information et les documents quels que soient leurs supports, et d'en faciliter le repérage et l'exploitation par les utilisateurs finaux. Adresse électronique : http:// www.ATD-doc.com. 


\section{BRIGITTE GUYOT}

Brigitte Guyot mène des recherches en sociologie de l'information et suit des projets qui mettent en place des systèmes d'information (plates-formes collaboratives, Intranet) dans des collectivités territoriales et entreprises privées. Elle y étudie à la fois le rôle du management et la place des utilisateurs. Elle étudie aussi la place d'Internet en milieu universitaire et dans les PME de pays en développement. A publié « dynamiques informationnelles dans les organisations » (Hermès-Lavoisier, 2006). Adresse électronique : http://www.brigitte-guyot.com. 